H. NAKAZATO

KODAI MATH. J.

3 (1980), 380-384

\title{
ON ODD TWO-DIMENSIONAL ICOSAHEDRAL GALOIS REPRESENTATIONS WITH SQUARE FREE CONDUCTOR
}

\author{
By Hajime Nakazato
}

\section{Introduction}

Let $\boldsymbol{Q}$ be an algebraic closure of the rational number field $\boldsymbol{Q}$, and let $G$ be the Galois group $\operatorname{Gal}(\overline{\boldsymbol{Q}} / \boldsymbol{Q})$. In [2], Deligne and Serre proved that the Mellin transform of a normalized new form of weight 1 with character is the Artin $L$ function of a continuous two-dimensional representation of $G$. The purpose of this paper is to investigate such representations of $G$.

Let

$$
\rho: G \longrightarrow \mathrm{GL}(2, C)
$$

be a two-dimensional continuous complex linear representation of $G$, and let

$$
\varepsilon=\operatorname{det}(\rho): G \longrightarrow \mathrm{GL}(1, C)=C^{\times} .
$$

Let $c \in G$ be a "complex conjugate", or Frobenius at infinity. We say that $\rho$ is odd if $\varepsilon(c)=-1$. Let $N$ be the (Artin) conductor of $\rho$. The conductor of $\varepsilon$ divides $N$ (cf. [4]). Let $\chi$ be a character of a group $H ; \chi: H \rightarrow C^{\times}$. Then we say that $\chi$ has order $n$ if the image of $\chi$ has order $n$, and we denote it by; ord $(\chi)=n$.

Let $\tilde{\rho}$ be the projective representation of $G$ attached to the linear representation $\rho$ of $G$;

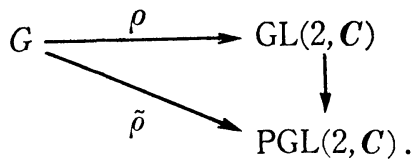

The image of $\tilde{\rho}$ is a finite subgroup of $\operatorname{PGL}(2, \boldsymbol{C})$. Hence it is one of the followings ;

1) cyclic groups,

2) dihedral groups,

3) the alternating groups $A_{4}, A_{5}$, and the symmetric group $S_{4}$.

We say that $\rho$ is of type $A_{4}$ (resp. $S_{4}, A_{5}$ ) if $\tilde{\rho}(G) \cong A_{4}$ (resp. $S_{4}, A_{5}$ ) (cf. [6]), and that $\rho$ is icosahedral if it is of type $A_{5}$.

Received July 17, 1979 
Our main result is the following theorem.

THEOREM I. Let $\rho$ be an odd continuous two-dimensional linear representation of $G$ with conductor $N$. Suppose that $\rho$ is of type $A_{5}$, and that $N$ is square free. Then the order of the image of $\rho$ is $240,720,1200$ or 3600.

Remark. In $\S 8$ of [6], Serre has remarked that if $N$ is a prime then the order of the image of $\rho$ is 240 . See Remark 1 in $\S 2$ and Proposition in $\S 3$. Moreover we see that if $N$ is a product of two distinct primes then the order of the image of $\rho$ is 240,720 or 1200 . See Remark 2 in $\S 2$.

\section{§1. Local theory}

Let $N=\prod_{p} p^{m(p)}$, and let $I_{p} \subset G$ be an inertia subgroup of a prime $p$.

Lemma 1. Suppose that $m(p)=1$. Then $\rho$ is tamely ramified at $p$. Moreover there exists a one-dimensional representation $\phi \neq \imath$ of of $I_{p}$ such that $\left.\rho\right|_{I_{p}}$ is isomorphic to the representation $\phi \oplus$ id of $I_{p}$. We have:

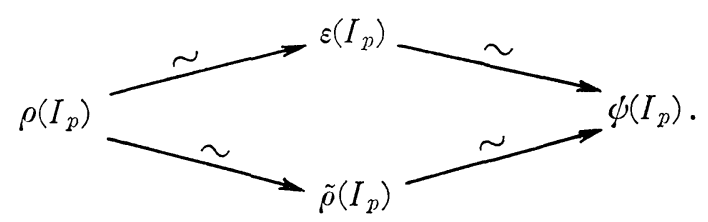

Proof. Let $D_{p} \supset I_{p}$ be the decomposition group of the place $v$ of $\overline{\boldsymbol{Q}}$ such that $I_{p}$ is the inertia group of $v$. We identify $D_{p}$ with the Galois group $\operatorname{Gal}\left(\overline{\boldsymbol{Q}}_{p} / \boldsymbol{Q}_{p}\right)$ of an algebraic closure $\overline{\boldsymbol{Q}}_{p}$ of the $p$-adic number field $\boldsymbol{Q}_{p}$. Let $K \subset \overline{\boldsymbol{Q}}_{p}$ be the fixed field of $\operatorname{Ker}\left(\left.\rho\right|_{D_{p}}\right)$. Then we have $\operatorname{Gal}\left(K / \boldsymbol{Q}_{p}\right) \cong \rho\left(D_{p}\right)$. Let $H=\mathrm{Gal}\left(K / \boldsymbol{Q}_{p}\right)$, and let $\rho^{\prime}: H \rightarrow \mathrm{GL}(2, \boldsymbol{C})$ be the representation of $H$ induced by $\left.\rho\right|_{D_{p}}$. Let $V$ be a representation space of $\rho^{\prime}$, and let $G_{\imath}(i \geqq 0)$ be the corresponding ramification groups ( $G_{0}$ being the inertia group) in $H$. By the formula of Artin conductor, we have

$$
m(p)=\sum_{i=0}^{\infty} \frac{\left|G_{\imath}\right|}{\left|G_{0}\right|} \operatorname{codim}\left(V^{G_{i}}\right)
$$

(cf. [4], [5]). If $\rho$ is not tamely ramified at $p$. Then we have: $G_{1} \neq\{\imath d\}$. Since $\rho^{\prime}$ is a faithfull representation, we have: $\left(\operatorname{codim}\left(V^{G_{0}}\right) \geqq\right) \operatorname{codim}\left(V^{G_{1}}\right) \geqq 1$. Hence

$$
\begin{aligned}
m(p) & =\sum_{i=0}^{\infty} \frac{\left|G_{i}\right|}{\left|G_{0}\right|} \operatorname{codim}\left(V^{G_{i}}\right) \\
& \geqq \frac{\left|G_{0}\right|}{\left|G_{0}\right|} \operatorname{codim}\left(V^{G_{0}}\right)+\frac{\left|G_{1}\right|}{\left|G_{0}\right|} \operatorname{codim}\left(V^{G_{1}}\right) \\
& \geqq 1+\frac{\left|G_{1}\right|}{\left|G_{0}\right|} .
\end{aligned}
$$


So we have: $m(p) \geqq 2$. This contradicts the assumption that $m(p)=1$. The first assertion is proved.

Since $\rho$ is tamely ramified at $p, \rho\left(I_{p}\right)$ is a cyclic group. Hence there exist one-dimensional representations $\psi_{1}$ and $\psi_{2}$ of $I_{p}$ such that $\left.\rho\right|_{I_{p}}$ is isomorphic to $\psi_{1} \oplus \psi_{2}$. Considering the conductors of $\left.\rho\right|_{I_{p}}$ and $\phi_{1} \oplus \psi_{2}$, we have :

$$
\phi_{1}=i d \text { and } \phi_{2} \neq i d \text {, or } \phi_{1} \neq \imath d \text { and } \phi_{2}=\imath d \text {. }
$$

Therefore $\left.\rho\right|_{I p}$ is isomorphic to $\phi \oplus \imath d$ with $\phi \neq \imath d$. The proof is completed.

Remark. Let $N$ be square free. Then by Lemma 1 the conductor of $\tilde{\rho}$ (see $\S 6$ of [6]) is $N$ and the conductor of $\varepsilon$ is $N$.

\section{$\S 2$. The order of $\varepsilon$}

THEOREM II. Let $\rho$ be an odd continuous two-dimensional representation of $G$ with conductor $N$, and put $\varepsilon=\operatorname{det}(\rho)$. Suppose that $N$ is square free. Then we have the followings.

i) The order of $\varepsilon$ is 6 , if $\rho$ is of type $A_{4}$.

ii) The order of $\varepsilon$ is 2, 4, 6 or 12 , if $\rho$ is of type $S_{4}$.

iii) The order of $\varepsilon$ is $2,6,10$ or 30 , if $\rho$ is of type $A_{5}$.

Remark 1. If $N$ is a prime. Then the followings were obtained in Theorem 7 of [6].

i) There exists no representation of type $A_{4}$.

ii) The order of $\varepsilon$ is 2 or 4 , if $\rho$ is of type $S_{4}$.

iii) The order of $\varepsilon$ is 2 , if $\rho$ is of type $A_{5}$.

Remark 2. Suppose that $N$ is a product of two distinct primes. Then we have the followings.

i) The order of $\varepsilon$ is 6 , if $\rho$ is of type $A_{4}$.

ii) The order of $\varepsilon$ is $2,4,6$ or 12 , if $\rho$ is of type $S_{4}$.

iii) The order of $\varepsilon$ is 2,6 or 10 , if $\rho$ is of type $A_{5}$.

To obtain Theorem II, we use the following lemma.

LEMMA 2. The Galois group $G$ is generated, in the sense of topological groups by all conjugates of inertia subgroups of all primes.

Proof. Let $G^{\prime}$ be the subgroup of $G$ generated by all conjugates of inertia subgroups of all primes. Then the fixed field of $G^{\prime}$ is unramified over $\boldsymbol{Q}$. Hence we have: $G=G^{\prime}$, by Minkowski's Theorem (cf. [1], Chap. 2, Sec. 6, Problem 4, p. 129).

Proof of Theorem II. Let $n_{p}=\operatorname{ord}\left(\left.\varepsilon\right|_{I_{p}}\right)$ for each prime $p \mid N$, and let $n=\operatorname{ord}(\varepsilon)$. Then $n$ is even since $\rho$ is odd. Let $\zeta$ be a primitive $n$-th root of 
unity. For a subset $A$ of a group $H$, let $\langle A\rangle$ be the subgroup of $H$ generated by $A$. By Lemma 2, we have

$$
\varepsilon(G)=\left\langle\varepsilon\left(I_{p}\right)\right| \text { all primes } p|N\rangle .
$$

So we have $\langle\zeta\rangle=\left\langle\zeta^{n / n} p\right|$ all primes $p|N\rangle$. Hence there exist integers $a_{p}, p \mid N$, such that

$$
1=\sum_{p \nmid N} a_{p} \frac{n}{n_{p}} .
$$

Since $\varepsilon\left(I_{p}\right) \cong \tilde{\rho}\left(I_{p}\right), \varepsilon\left(I_{p}\right)$ is isomorphic to a cyclic subgroup of $\tilde{\rho}(G)$ for each $p \mid N$. For each $p \mid N, n_{p}$ is 2 or 3 (resp. 2, 3 or $4 ; 2,3$ or 5) if $\rho$ is of type $A_{4}$ (resp. $\left.S_{4} ; A_{5}\right)$. Hence there exist non-negative integers $a, b$ and $c$ such that $n=2^{a} 3^{b} 5^{c}$. Moreover noting that $n$ is even, we have the followings.

i) $a=1,0 \leqq b \leqq 1, c=0$, if $\rho$ is of type $A_{4}$.

ii) $1 \leqq a \leqq 2,0 \leqq b \leqq 1, c=0$, if $\rho$ is of type $S_{4}$.

iii) $a=1,0 \leqq b \leqq 1,0 \leqq c \leqq 1$, if $\rho$ is of type $A_{5}$.

Hence we have:

i) $n$ is 2 or 6 , if $\rho$ is of type $A_{4}$.

ii) $n$ is $2,4,6$ or 12 , if $\rho$ is of type $S_{4}$.

iii) $n$ is $2,6,10$ or 30 , if $\rho$ is of type $A_{5}$.

By the same reason as in the proof of Theorem 7, pp. 276-277, in $\S 8$ of [6], if $\rho$ is of type $A_{4}$ then $n$ is 6 . The proof is completed.

\section{$\S 3$. The proof of Theorem 1}

The following proposition and Theorem II imply Theorem I.

Proposition. Let $\rho$ be an odd continuous two-dimensional linear representation of $G$, and let $n$ be the order of $\operatorname{det}(\rho)$. Suppose that $\rho$ is of type $A_{5}$. Then the order of the image of $\rho$ is $120 \mathrm{n}$.

Proof. Let $H=\operatorname{Ker}\left(\rho(G) \stackrel{\operatorname{det}}{\longrightarrow} C^{\wedge}\right)$. Then $(\rho(G): H)=n$. Let $Z$ be the subgroup of $\mathrm{GL}(2, C)$ consisting of all scalar matrices, and put $Z_{0}=\rho(G) \cap Z$. Then $\rho(G) / Z_{0} \cong A_{5}$. The subgroup $H$ is a normal subgroup of $\rho(G)$. Hence from the following commutative diagram;

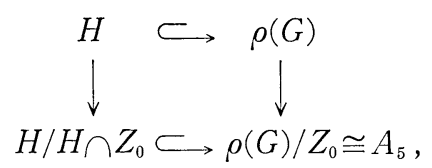

we see that $H / H \cap Z_{0}$ is a normal subgroup of $A_{5}$. Therefore we have $H / H \cap Z_{0}$ $\cong A_{5}$, since $A_{5}$ is a simple group. By the definitions of $H$ and $Z_{0}$, we have two cases ; 

a) $H \cap Z_{0}=\left\{\left(\begin{array}{ll}1 & 0 \\ 0 & 1\end{array}\right)\right\}$,
b) $H \cap Z_{0}=\left\{ \pm\left(\begin{array}{ll}1 & 0 \\ 0 & 1\end{array}\right)\right\}$.

In the case a), we have $H \cong A_{5}$. Since $H$ is a subgroup of $\operatorname{GL}(2, C)$. This contradicts the classification of finite subgroups of $\mathrm{GL}(2, \boldsymbol{C})$ (cf. $\$ 26$ of [3]). Therefore the case a) does not occur.

In the case b), the order of $H$ is 120 . Hence the order of $\rho(G)$ is $120 n$. The proof is completed.

Remark. In this proposition, we make no assumption on the conductor of $\rho$.

\section{REFERENCES}

[1] Z.I. Borevich \& I.R. Shafarevich: Number Theory. Academic Press, New York and London, 1966.

[2] P. Deligne \& J-P. Serre: Formes modulaires de poids 1. Ann. sci. E.N.S. 7, 507-530 (1974).

[3] L. Dornhoff: Group Representation Theory, Part A, Marcel Dekker, Inc., New York, 1971.

[4] I. MARTinet : Character theory and Artin L-functions, Proceedings of a conference at Durham, 1977.

[5] J-P. Serre: Corps locaux. Hermann, Paris, 1968.

[6] J-P. SERRE: Modular forms of weight one and Galois representation. Proceedings of a conference at Durham, 1977.

Department of Mathematics,

Tokyo Institete of Technology. 